\title{
CLOPS: A DSL for Command Line Options
}

\author{
Mikoláš Janota, Fintan Fairmichael, Viliam Holub, \\ Radu Grigore, Julien Charles, Dermot Cochran, and Joseph R. Kiniry \\ School of Computer Science and Informatics, \\ Lero - The Irish Software Engineering Research Centre, and \\ The Complex and Adaptive Systems Laboratory (CASL), \\ University College Dublin, Ireland
}

\begin{abstract}
Programmers often write custom parsers for the command line input of their programs. They do so, in part, because they believe that both their program's parameterization and their option formats are simple. But as the program evolves, so does the parameterization and the available options. Gradually, option parsing, data structure complexity, and maintenance of related program documentation becomes unwieldy. This article introduces a novel DSL called CLOPS that lets a programmer specify command line options and their complex inter-dependencies in a declarative fashion. The DSL is supported by a tool that generates the following features to support command line option processing: (1) data structures to represent option values, (2) a command line parser that performs validity checks, and (3) command line documentation. We have exercised CLOPS by specifying the options of a small set of programs like 1s, gzip, and svn which have complex command line interfaces. These examples are provided with the Open Source release of the CLOPS system.
\end{abstract}

\section{Introduction}

A boiling frog is a well known phenomenon in software development. Just as a frog meets its fate in water that is slowly heated, so do software projects become unmanageable as their complexity gradually increases.

Processing of command line options is not an exception. The first few versions of a program have small number of options that are easy to process and do not have many dependencies between them.

No wonder then, that many programmers, with famous last words "this is easy" on their lips, opt for custom code for option processing. As the number of the program's options and their inter-dependencies grows, option processing code gets bloated and diverges from its documentation.

This article suggests an alternative by providing a DSL that enables programmers to explicitly, declaratively capture options and their inter-dependencies. Furthermore, a tool is provided that produces documentation, code for command line processing, and data structures. The data structures produced represent the values of the options and are automatically populated during parsing. 


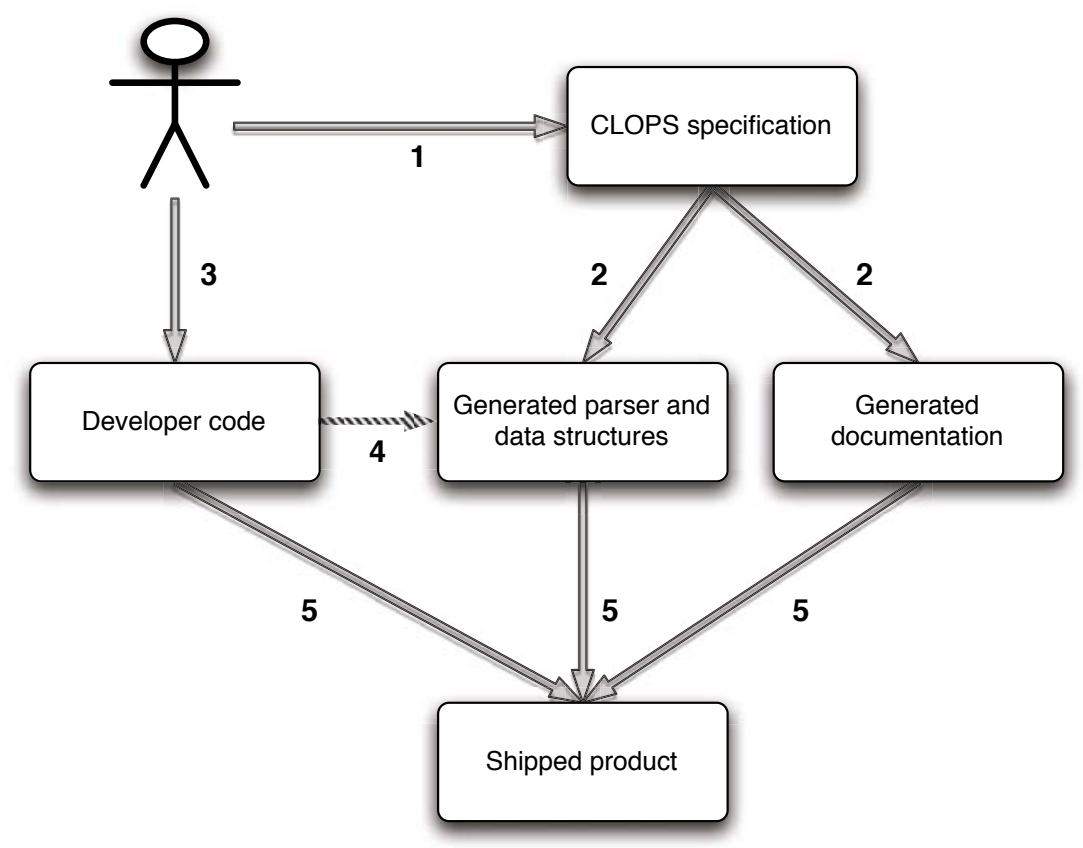

Fig. 1. Developer usage scenario

The observations described above shaped the language proposed in this article. On the one hand, simple option sets must be easy to write when the program is in its early stages. But on the other hand, a set of options must be easy to evolve and even complicated option sets must be supported.

There are two types of users involved in using CLOPS - the developer who specifies the command line interface and integrates the command line parser with the main program body, and the end-user who interacts with the final product by executing it and providing options on the command line. Fig. 1 gives a diagrammatic representation of a developer's usage scenario for creating a command line program using CLOPS.

The developer describes the command line of the tool in the CLOPS DSL(1). This description is used by the CLOPS tool to produce code and documentation(2). The code is used as a library(4) by the developer to parse the command line(3). The result of parsing is a set of data structures (options) that can be easily queried. The documentation is typically presented to the enduser(5).

The end-user is typically not aware that the developer's tool uses CLOPS. This is despite the fact that CLOPS parses the command line, provides many of the error messages, and generates the documentation that the user consults (such as a man page and the usage message printed by the program itself). 
This article contains several core contributions:

- We identify the main concepts seen in command line processing and describe their interdependencies (Section 4).

- We propose a novel DSL called CLOPS for describing command line option interfaces. Its syntax and semantics are described in Section 5.

- We provide a tool (its implementation is discussed in Section 6) that reads in a description in this language and generates multiple artifacts:

- a Java implementation of the command line parser,

- documentation, and

- data structures that are used to store the result of parsing.

- Section 7) summarizes results of applying CLOPS to well-known programs like 1s; interesting cases are discussed and the CLOPS description is compared to existing implementations.

- Section 8 is a summary of experience of one of the authors that have applied CLOPS in projects he has been working on.

Section 9 elaborates how our research relates to other parts of Computer Science, namely Software Product Lines. Section 10 is an overview of other approaches tackling command line processing and Section 11 provides pointers for further research and improvements. The upcoming section sets up the ground for further discussion and is followed by a light-weight exposure to CLOPS DSL in Section 3.

\section{Background}

Command line utilities are widespread in the software community and hence there is no need to go into great detail about them. Nevertheless, several properties of command line options that will be important for later discussion must be reviewed.

A program is run from a command line with a list of options that affect its behavior. For instance, the program is lists files in a multi-column format if run with the option $-\mathrm{C}$ and lists files one-per-line if run with the -1 option.

This means that the invoked program has to process the options provided on the command line and store the result of that analysis into its state in some fashion.

Obviously, the behaviors of these particular options, $-\mathrm{C}$ and -1 , cannot be put into effect at the same time. We say that there is a dependency between these two options.

As a consequence, $1 \mathrm{~s}-\mathrm{C}-1$ results in the same behavior as if only -1 was provided and vice versa, according to the principle last one wins. For such dependency we say that the options override one another. There are several kinds of dependencies with varying resolution strategies.

Another common pattern seen in command line arguments is option arguments. To give an example, the program tail prints the last $x$ lines of a given input and is typically invoked with tail $-\mathrm{n} x$ filename. This means that option processing needs to make sure that $\mathrm{x}$ is indeed a number, and produce an error otherwise, and convert its textual representation into a numeric one. Often, 


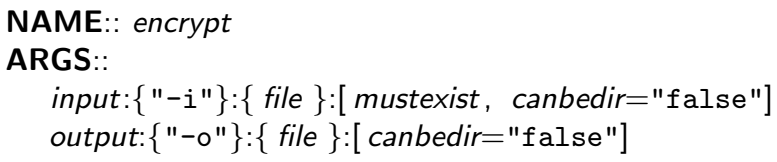

FORMAT:: input output;

Listing 1. A simple CLOPS description of an encrypting program

an argument has a default value. If tail is invoked without the $-\mathrm{n}$ option, for instance, $\mathrm{x}$ is assumed to have value 10 .

The set of options supported by a certain program represent its (textual) user interface. Hence, it is important for a program's options to be well-documented as a program's users cannot be expected to read its source code. In UNIX-like systems documentation is maintained in the database of man(ual) pages. As stated before, one of the objectives of this work is to keep a program's documentation consistent with its behavior.

\section{CLOPS Exposure}

Let us imagine we are writing a program which encrypts a given file. As we want to avoid problems with weirdly-named files (such as starting with the hyphen), we introduce one-argument options -i filename and -o filename to enable the user to specify the input and the output file, respectively.

Listing 1 is how we write it in CLOPS. The list under ARGS provides the possible command line options identified as input and output. For both of the options it specifies that they accept file-names as values. Additionally, the file given as input must exist and none of the files can be a directory.

The FORMAT is a regular expression that tells us that both input and output must be specified and input comes first.

The parser generated according to this CLOPS description will accept command lines such as $-i$ foo -o bar, provided that the file foo exists.

After a while we get bored with this restrictive form and we decide to enable the user not to specify the output file as the program will derive the name from the name of the input file. Additionally, our colleague who has been using our tool is annoyed by all the debugging messages that are meaningless to him and we introduce the quiet mode. Since we have our first end-user, we add textual descriptions so we can generate simple documentation. The result can be seen in Listing 2. Note that for quiet we have not specified any type, which is the same as writing quiet:\{"-q" $\}:\{$ boolean\}.

\section{Concepts}

The previous sections highlighted several common patterns that are seen in command line interfaces. Namely, we have different types of options, options depend on one another, and the order of options on the command line is important. 


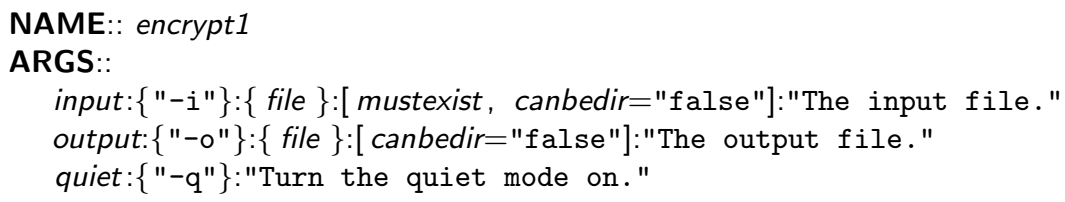

FORMAT:: quiet? input output?;

Listing 2. Extended version of the encrypting program

What follows is a concept analysis of this domain. Each of these concepts is later reflected in the CLOPS DSL.

Options. The command line interface of a particular program focuses on a set of options. Each option has a type such as string, number, or file. For parsing purposes, each option is associated with a set of strings (possibly infinite) that determines how the option is specified on the command line. For instance, for the command head, any string of the form -n NUMBER specifies the number-oflines option. For a given option, we will call these strings match strings. Last but not least, an option has a documentation string for end-user documentation purposes.

Values. Parsing a command line results in an internal representation of that particular command line. We will refer to this result as option values. As the program executes, these values control its behavior. Each option can be set to a value that must agree with the option's type. For example, the value of the number-of-lines option seen above, is an integer.

Format. The ordering of options provided on the command line is often important. Different orderings may have different meanings to the program, and sometimes a different ordering of the same options is not valid. The format specifies all valid sequences of options.

We should note that the format not only determines whether a sequence of options is valid, but plays an important role in parsing. For instance, in the case of the version control system svn, one can write svn add add, where the first occurrence of the string add means "add a file to the repository" and the second occurrence means that the file to be added is called add. The parser is able to make this distinction as svn's format specifies that a command must precede a filename and only one command use is permitted.

Validity. Only certain combinations of option values are valid. A function that determines whether a given combination of the values is valid or not is called a validity function. Validity functions are used to enforce constraints on options, for instance ensuring that two mutually exclusive options are not both enabled. Whenever an end-user specifies option values that fail validity testing, the command line parser produces an appropriate error message. 
Rules. A change in the value of one option may affect values of other options. Such relationships are represented using rules that are triggered during parsing to change the value of other options. More specifically, a rule consists of: a trigger, telling us at which point of the format it may fire; a guard, a condition for firing; and an effect, the action executed when the guard holds. Rules are used to realize, among other features, overriding. For instance, in ls, the option -1 triggers a rule that sets $-\mathrm{C}$ to false, and vice versa.

Command line string. An option is associated with a set of strings that state how that particular option is specified on the command line. However, each command line specifies multiple options.

In practice, a command line input is represented as a list of strings. A special character (denoted here by ${ }_{-}$) is appended to each of these strings and then the strings are concatenated to produce the command line string. This string will be used as the input to the parser.

Parsing partitions the given command line string into substrings, each corresponding to a different option. For instance, ls run with the command line

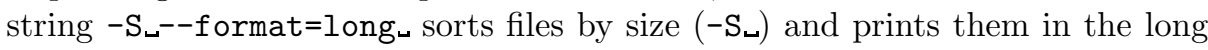
format (--format=long - $_{\text {) }}$

Usually options come on the command line separately, therefore the match strings typically contain the delimiter character. For instance, the ls command's --format option matches on --format=FORMATSPECIFIER, where the FORMATSPECIFIER is one of across, commas, horizontal, long, single-column, verbose, and vertical.

In some programs, the individual match strings do not have to be delimited. For instance, ps xa is equivalent to ps $\mathrm{x}$ a. This means that the option $\mathrm{x}$ matches on the character $\mathrm{x}$ optionally followed by. . These patterns motivated us to represent a command line input as a single string as we can treat such options uniformly.

\section{DSL}

We define the CLOPS domain specific language by giving its syntax and its semantics.

\subsection{Syntax}

Fig. 2 gives an overview of the CLOPS syntax1. The meta-symbols used in the right hand side of productions $\left({ }^{*},+, ?, \mid\right)$ have the usual meaning (zero or more times, one or more times, optional, alternative). Parentheses are also meta-symbols. (We omit the literal round parentheses from the grammar for clarity.) Terminals are typeset in bold. Nonterminals are typeset in roman if their productions are present, and are typeset in italics if their productions are missing.

\footnotetext{
${ }^{1}$ See also http://clops . sourceforge.net/clops/grammar.html
} 


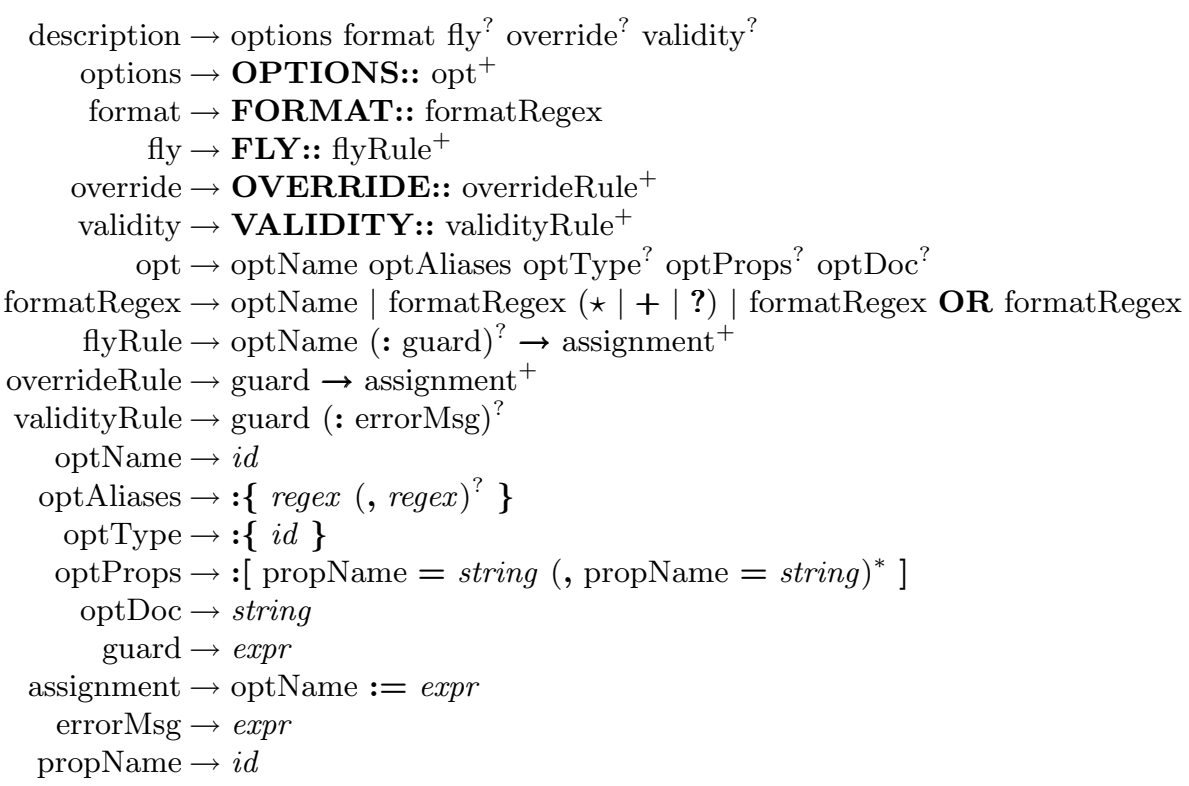

Fig. 2. Syntax of the CLOPS DSL

There are four nonterminals not described in the grammar: id, string, regex, and expr. An id is a Java identifien?2. A string is a Java string literal. A regex is a Java string literal $s$ that does not cause an exception when used in an expression Pattern.compile(s). An expr is a side-effect-free Java expression whose type is constrained, as discussed later.

A command line parser description in CLOPS consists of one or more option declarations, a format declaration, and, optionally, rule declarations. The informal meaning of each of these syntax sections is summarized through a series of examples below. A formal semantics is given in the next section.

Example 1: A Prototypical Option Declaration Here is an example of an option declaration:

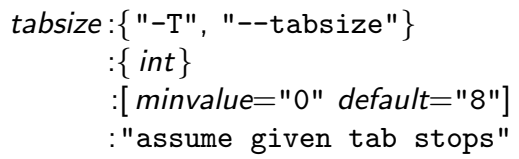

The name of the option is tabsize, it has aliases "-T" and "--tabsize", its type is int, and its documentation string is "assume given tab stops". Values are given to the option properties minvalue and default. The type int is not a Java type, but is an option type. The built-in types include string, file, and boolean, which is the default option type. The complete set of built-in option types is given later in Table 1, and Section 6 discusses how the type set is extended.

${ }^{2}$ The option type is allowed to contain -, unlike Java identifiers. 
The match strings of the option tabsize are the words of the regular lan-

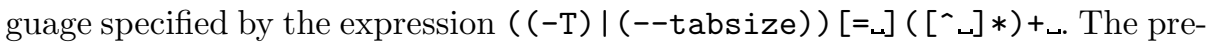
fix $((-\mathrm{T}) \mid(--$ tabsize $))$ is built from the aliases, while the suffix $\left[=_{-}\right]\left(\left[{ }^{*}\right] *\right)+^{-}$ is automatically defined by the option type. The suffix is overridden for any option type using the property suffixregexp .

For another example, consider again the command head. This command permits the user to write $-\mathrm{n}$ NUMBER but also a shorter version -NUMBER. To specify this shorthand syntax, the suffix is $(\backslash \backslash d)+\_$(non-zero number of digits followed by the delimiter), and the prefix - (a hyphen).

Example 2: Format Strings using Options. A convention many UNIX tools follow is to consider everything on a command line that does begin with a hyphen character (-) as not being a filename. All file names that do start with a hyphen must appear after the special option --. This behavior is captured in the format string:

(Flag OR File)* (HyphenHyphen HyphenFile*)?

Here Flag, File, HyphenHyphen, and HyphenFile are option names. Section 6 introduces option groups, which make formats easier to write.

Example 3: Fly Rules. Fly rules are used, among other purposes, to obtain the 'last wins' behavior discussed earlier with regards to the 1s command. Two fly rules that describe this interface are:

HyphenOne $->$ HyphenC $:=$ false
HyphenC $->$ HyphenOne $:=$ false

Validity rules, such as the following, are used if an error is preferable:

HyphenOne? \&\& HyphenC? -> "Both -1 and $-C$ were given."

For fly rules, a trigger is specified via an option name on the left of the arrow. For override rules, the trigger is described similarly. All validity functions are triggered when parsing finishes.

The fly rule examples above do not specify a guard, so the guard defaults to true. The validity rule example contains an explicit guard and illustrates some convenient syntactic sugar. The API generated for each option consists of a method to query its value and a method to query if it was set at all. The option name used in an expression is desugared into a call to get the option's value, while the option name followed by a question mark is desugared into a call to check if the option was set. As expected, the (Java) type of the guard must be boolean. In the future we plan to allow JML [12] expressions to be used as guards.

The actions of fly rules are assignments. The left hand side of an assignment names an option, while the right hand side contains an expression. The expression must have a type convertible (according to Java rules) to the type of the option's value. Override rules behave the same fashion, but validity rules are different. As seen in the validity rule example, the action of the rule is not written as an assignment, but is instead implicit. In this case, the assigned option has the type string - list, and is used to collect error messages. 
The validity function is specified through validity rules. These rules are triggered after parsing has finished and, if their guard evaluates to true, the string on the righthand side is produced as an error.

\subsection{Semantics}

As previously mentioned, a CLOPS description is used to generate several artifacts, such as documentation and source code. The command line parser for the described options is the crux of these artifacts.

The mapping of the CLOPS description to a generated parser defines the DSL's semantics. The goal of this section is to provide an operational semantics of the command line parser for a given CLOPS description.

To be able to discuss a CLOPS description formally, we will assume that we have several mathematical objects that correspond to it.

Preliminaries. Assume the existence of a finite state automaton $\mathcal{A}$. Each transition of $\mathcal{A}$ is labeled with an option $o$ and a set of rules $R$. The notation $s \rightarrow^{o, R} s^{\prime}$ denotes that there is a transition from state $s$ to state $s^{\prime}$ in $\mathcal{A}$, labeled with the option $o$ and the set of rules $R$.

Legal sequences of options, as given by the CLOPS description, correspond to paths in the automaton that start in the starting state and end in an accepting state. The automaton is built in the usual way from the regular expression specified via the CLOPS format section.

A rule is a triple $(g, o, e)$ containing a guard, an option, and a side-effect-free value expression. The intuition behind the rules is that all the rules labeling a transition are triggered whenever that transition is taken. When a rule $(g, o, e)$ is triggered, its guard $g$ is evaluated. If the guard evaluates to true, then the expression $e$ is evaluated and the result is assigned to the value of the option $o$. We say that such a rule has fired.

The function parse computes a value for a given option from an input string. Hence, its type is Option $\times$ String $\rightarrow$ Value. The manner in which a String is converted to a Value is defined by the Option in question.

The function match tries to find a matching string of a given option among the prefixes of the command line string. Using the functional languages convention, its type is Options $\times$ String $\rightarrow$ Maybe String (). If the match is successful it returns the prefix on which the option matches, otherwise it returns null.

The option store is a partial function $V$ from options to their values. The function is partial as when an option has not been set, it has no value.

Details. Let us now proceed with the definition of the command line parser based on these mathematical objects (the automaton $\mathcal{A}$ and the functions parse and match).

The parsing of the command line string is a traversal of the automaton. At each stage of the parse the available transitions from the current automaton state represent the options that are valid for use at this point in the command line string. Each available option is checked to see if it matches at the current 
position of the command line string. If no option matches, we produce an error. If an option matches we take the transition for that option, fire the rules that label the same transition if their guards evaluate to true, and move forward in the command line string by the match length.

The rest of the section defines this traversal formally.

Some new notation is convenient. The notation $V[x \mapsto v]$ stands for a function that returns $V(y)$ if $x \neq y$ and $v$ otherwise. For the side-effect-free expression $e$ defined on the option values $V$, the notation $e(V)$ represents the value to which $e$ evaluates after substituting values for options.

The operational semantics of the command line parser is given as a transition function on the parser's states. A state of the parser is either an error state or a triple $(c, V, s)$, a command line string (see Section 4), a function from options to their values, and a state of the automaton $\mathcal{A}$, respectively.

For a state $(c, V, s)$ we define the following two auxiliary sets:

1. Let Legal be the set of options labeling a transition from $s$ in $\mathcal{A}$

$$
\operatorname{Legal}(s) \equiv\left\{o \mid\left(\exists s^{\prime}\right)\left(s \rightarrow^{o, R} s^{\prime}\right)\right\}
$$

2. Let Matched be a subset of Legal comprising options that match on $c$.

$$
\operatorname{Matched}(s, c) \equiv\{o \in \operatorname{Legal}(s) \mid \operatorname{match}(o, c) \neq \mathbf{n u l l}\}
$$

The parser transition goes from the state $(c, V, s)$ to the state $\left(c^{\prime}, V^{\prime}, s^{\prime}\right)$ if Matched contains exactly one option $o$ and $s \rightarrow^{o, R} s^{\prime}$. The command line $c^{\prime}$ is obtained from $c$ by stripping the prefix $\operatorname{Match}(o)$.

The values of the options are updated by computing the function Parse for $o$ and by triggering, in parallel, the rules labeling the transition being taken. This is formally written as follows.

Let $V^{\prime \prime}=V[o \mapsto \operatorname{parse}(\operatorname{match}(o, c))]$. Then $V^{\prime}$ is defined as

$$
V^{\prime}(p)= \begin{cases}e(V) & \text { if there is a single rule }(g, p, e) \in R \text { and } g\left(V^{\prime \prime}\right) \text { holds, or } \\ V^{\prime \prime}(p) & \text { there is no such a rule. }\end{cases}
$$

This covers the case when $\mid$ Matched $\mid=1$ and there are no conflicting rules. However, if $\mid$ Matched $\mid=1$, and there are at least two rules whose guards evaluate to true that assign different values to the same option, then $(c, V, s)$ goes to the error state.

If the set Matched is empty, there is no transition of $\mathcal{A}$ to be taken. If it contains more than one option, the parsing is ambiguous. Thus, if $\mid$ Matched $\mid \neq 1$ we also go to the error state.

The computation of the command line parser starts in a state with the command line string to be processed, an option value function not defined anywhere (not assigning any values), and the start state of the automaton.

Note: In Future Work we suggest how one could check statically that the size of the set Match is at most one. 


\section{Implementation}

Source code and builds of the implementation are freely available for download on the tool's websit 3 . There is also a user-oriented tutorial that details the first steps in running the tool and creating a CLOPS description. The tool has been implemented in Java and requires a Java Runtime Environment of at least version 5.0. Although we chose to write our implementation in Java, there is nothing to stop our approach from being implemented in other programming languages, as we do not rely on any language features that are unique to Java.

The overall functionality of the tool is as one might expect: it parses a specification provided in the CLOPS DSL and produces a command line parser with consistent documentation.

\subsection{Option Types}

Recall that when a user declares an option in the DSL, they must specify the type of the option. An option type determines the Java type used to represent the option's value and, consequently, the parsing mechanism used for the corresponding option. Table 1 lists the built-in option types.

In order to extend the CLOPS DSL, new option types may be implemented and registered for use. An option factory defines the known set of options. Thus, to extend the CLOPS system and provide additional types, a developer extends the default option factory to make the system aware of new developer-provided types. The implementation of new option types can extend the built-in types to reuse and refine their functionality, or one may be written from the ground up, so long as the Option interface is satisfied.

While parsing each option declaration, the DSL parser queries the option factory for the corresponding option type. The option factory provides the necessary details of an option type: the class representing the option at runtime, as well as the Java type that is used for the value of the option.

The information provided by the option factory about each option's type is used in the code generation phase. An interface is generated that provides access to the value for each option. For instance, for a string option, a getter method with return type String is created. For each option there is also a method with return type boolean that determines whether an option has been set at all.

Each option type has a mechanism for indicating the properties that it accepts. When parsing a given input specification, we can check that the option will actually accept all provided properties. Assuming there are no problems, code is generated that sets the option properties to their specified values during the initialization phase.

\footnotetext{
3 http://clops.sourceforge.net/
} 
Table 1. Built-in option types and properties. Abstract types are typeset in italics. $T$ is the type of the concrete option's value.

\begin{tabular}{llll}
\hline Name & $\begin{array}{l}\text { Inherits } \\
\text { from }\end{array}$ & Java type & Properties \\
\hline basic & None & $T$ & default[T], suffixregexp[string] \\
\hline boolean & basic & boolean & allowarg[boolean] \\
\hline counted-boolean basic & int & $\begin{array}{l}\text { countstart[int], countmax[int], } \\
\text { warnonexceedingmax[boolean], } \\
\text { erroronexceedingmax[boolean] }\end{array}$ \\
\hline string & basic & String & \\
\hline string-regexp & string & String & regexp[string] \\
\hline string-enum & string & String & choices[string], casesensitive[boolean] \\
\hline int & basic & int & minvalue[int], maxvalue[int] \\
\hline float & basic & float & minvalue[float], maxvalue[float] \\
\hline file & basic & File & $\begin{array}{l}\text { canexist[boolean], mustexist[boolean], } \\
\text { canbedir[boolean], mustbedir[boolean], } \\
\text { allowdash[boolean] }\end{array}$ \\
\hline list & basic & List $\langle$ T $\rangle$ & allowmultiple[boolean], splitter[string] \\
\hline string-list & list & List $\langle$ String $\rangle$ \\
\hline file-list & list & List $\langle$ File $\rangle$ & $\begin{array}{l}\text { canexist[boolean], mustexist[boolean], } \\
\text { canbedir[boolean], mustbedir[boolean], } \\
\text { allowdash[boolean] }\end{array}$ \\
\hline & & & \\
\hline
\end{tabular}

\subsection{Option Groups}

A tool's format string sometimes grows quite long and is consequently difficult to understand and maintain due to its sheer number of options, independent of their dependencies. For this reason, we allow the options to be grouped.

An option group is defined by specifying a unique identifier paired with a list of options and/or other groups that are contained in the group. Then, when the identifier for a group is used in a format string, its definition is recursively expanded as a set of alternatives. Of course, these are hierarchical groups, and thus must be acyclic. We have found that appropriate use of option groupings make format strings much more concise, understandable, and more easily maintained.

There are many differing styles of documentation used to provide information to the end-user on the command line options available for a given tool. For this reason we have leveraged a powerful and mature open-source templating library, the Apache Velocity Project's templating engine [15], to produce documentation from the information contained within a CLOPS description. We provide several built-in templates for documentation generation, and if a developer requires a different style for their documentation, they can modify an existing template, or create their own. 


\section{Experiments}

In order to challenge CLOPS and to measure its effectiveness, several popular programs that have interesting option sets were described using the CLOPS system. Programs that are difficult or impossible to accurately describe using existing command line parsing tools without resorting to a large amount of custom parsing code were explicitly chosen.

Sources of information as to the semantics of a given tool's command line interface include manual pages, source code, command line help (e.g., tool --help), as well as trial-and-error with interesting option combinations.

We found describing these tools a useful exercise that led to many adjustments to our design and implementation. The fact that we were successfully able to describe their interfaces emphasizes the power and flexibility of our approach.

Additionally, even though all these programs are used by a large number of people, several inconsistencies were discovered in their documentation, and between their documentation and implementation.

Obviously, using the generated parser guarantees consistency between documentation and implementation. Moreover, the examples discussed in previous sections show that a systematic approach to recording options often highlights inconsistencies in their design, as well as their documentation.

\section{$7.1 \quad$ ls}

The program Is lists files found in the file-system, is frequently used and many options influence which information is displayed and in what format. The GNU implementation of Is comes with 56 options.

The options we have found the most interesting are options determining the format of the output. There is an option format, with the enumeration domain, where each of the enumeration values corresponds to a boolean option. The following snippet from the manual page shows how.

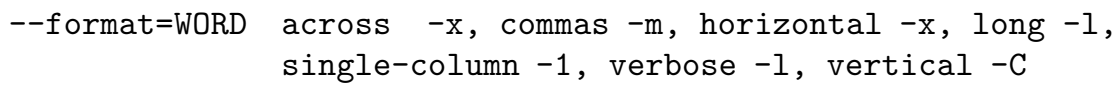

This means that invoking $1 \mathrm{~s}-\mathrm{C}$ should be the same as $1 \mathrm{~s}--$ format=vertical; ls --format=horizontal as ls -x etc.

This design is clearly a hotbed of inconsistencies and we have found one. The option format can have only one value at a time, i.e., if defined on the command line multiple times, the last one wins. According to the POSIX standard, however, the option -1 should be assumed whenever -1 is given 4 . As the GNU implementation follows the standard, the call $1 \mathrm{~s}-1-1$ results in a different output than is --format=long --format=single-column. The former corresponds to Is -1 while the latter to $1 \mathrm{~s}-1$. This means that the value single-column does not have the same effect as the option -1 and the documentation is inaccurate.

4 http://www .opengroup.org/onlinepubs/000095399/utilities/ls.html 
Interestingly enough, the BSD implementation of ls does not follow the POSIX standard as $1 \mathrm{~s}-1-1$ results in the equivalent of 1 s -1 , i.e., the options -1 and -1 are regarded as distinct options which override one another.

We believe that situations similar to the format option arise quite often: as the program evolves, new options are added (like -1) and at some point there is too many that deal with a similar issue that an enumeration option is introduced to unify them. If that is the case, maintaining backward compatibility is difficult.

We should note that the enumeration format does appear only in the GNU implementation.

How does CLOPS help? Rules let us specify the equality between options. The following CLOPS description snippet relates format's value "commas" and the boolean option commas.

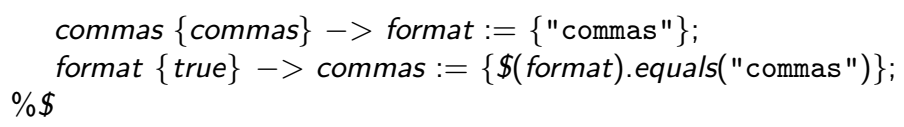

The first identifier in the rules is the triggering option followed by a condition and an action. The first rule specifies that if commas is set to true, the format's value is set to the value "commas". The second rule says that whenever the value of format changes, commas is set to true iff format's value is "commas".

Note that this specification is not quite symmetric, as it does not specify what should happen when commas is set to false. In the current solution we disallow the user to set the option explicitly to false as the following would result in an inconsistent state ( $-\mathrm{m}$ is a identifier of the option commas).

--format=commas $-\mathrm{m}=\mathrm{fal}$ se

Alternatively we could unset the option format if the option representing the current value is set to false. Not enabling boolean options to be set to false is seen in the GNU implementation.

Writing up the rules in this fashion clarifies which options correspond to one another.

The source code processing the options in the GNU implementation has 327 non-blank lines of code. The CLOPS description including the documentation has 246 non-blank lines.

\section{2 gzip}

The program gzip compresses files using Lempel-Ziv coding. The most interesting part of the command line interface for this tool is the compression level. The compression level has a value in the range 1 to 9 and defaults to 6 . The compression level is specified on the command line by prepending the number with hyphen ( -4 etc.) or by the predefined human-friendly aliases --fast for -1 and --best for -9 . In CLOPS, the compression level would be defined as an integer parameter:

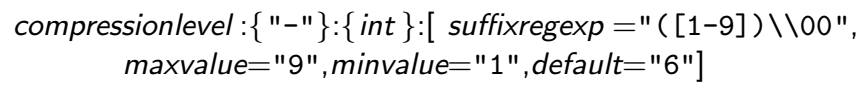


In other words, we set the option name to - with a suffix of one digit followed by an option separator. Compression level aliases are defined as regular options with fly rules setting the compression level:

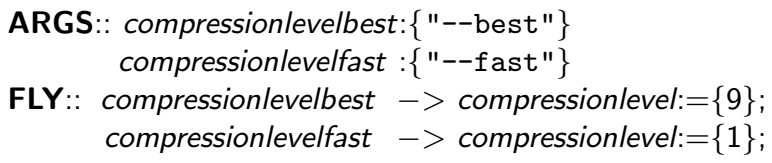

Although such a construction is simple and sufficient, there is a partial inconsistency when we use multiple options setting the compression level: for example, specifying --best --fast would correctly set the compression level to 1 , but leave the compressionlevelbest parameter set. Similarly, we would expect option compressionlevelbest to be set to true when -9 is provided. We can fix this with additional set/reset fly rules:

FLY::

compressionlevelbest $->$ compressionlevel: $=\{9\}$, compressionlevelfast $:=\{$ false $\}$; compressionlevelfast $->$ compressionlevel $:=\{1\}$, compressionlevelbest $:=\{$ false $\}$; compressionlevel $\{\$($ compressionlevel ). equals("9")\} $\rightarrow>$ compressionlevelfast $:=\{$ false $\}$, compressionlevelbest $:=\{$ true $\}$; compressionlevel $\{\$($ compressionlevel $)$. equals $(" 1 ")\}->$ compressionlevelfast $:=\{$ true $\}$, compressionlevelbest $:=\{$ false $\} ;$

The level of verbosity is increased with the verbose option -v. Using -v multiple times increases the verbosity level up to a maximum of three. In CLOPS we have a counted-boolean option that has type integer, that increases its count each time it is used. Gzip also has a quiet option - $\mathrm{q}$ that turns off some output. Using the quiet option resets the verbosity level to zero, and using the verbose option turns off quiet. To achieve this in CLOPS we can define verbose and quiet as the following:

\section{ARGS::}

verbose: $\{$ "-v","--verbose" $\}:\{$ counted-boolean $\}:[$ countmax="3", warnonexceedingmax] quiet: $\{$ "-q", "--quiet" $\}$

FLY::

quiet $->$ verbose: $=\{0\}$;

verbose $->$ quiet: $=\{$ false $\}$;

\section{Testimony}

One of the authors (Julien Charles), who joined the project later than the other authors, applied CLOPS to three projects he has been working on. The following text summarizes the experience he gained. As each of the projects was in a different stage of maturity it demonstrates three different approaches to the use of CLOPS as well as a guide to get a better understanding of the tool.

All the tools mentioned in this section are part of the Mobius tool suit 5 and are written in Java. The task was to (1) replace an existing complete front-end

5 Their source code can be obtained at http://mobius.ucd.ie 
ARGS::

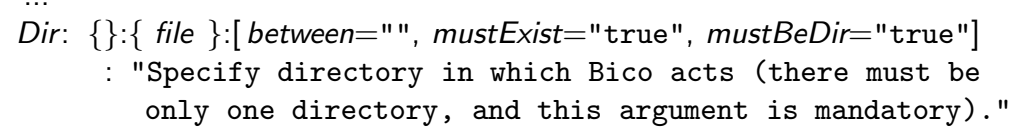

Clazz: \{\}$:\{$ string - list $\}:[$ between $="$ ", argumentshape $="[\mathrm{a}-\mathrm{zA}-\mathrm{Z}]+"$.

: "Generate also the file for the specified classes, bico must be able to find them in its class path."

\section{FORMAT::}

Help | (Dir (Misc| Type | Clazz)*);

Listing 3. Solution to the first problem

with CLOPS, (2) apply CLOPS to a tool with an unclearly defined list of options, and (3) apply the CLOPS methodology to a tool with no front-end yet.

\subsection{Replacing an Already Complete Option Handler}

The first encounter with CLOPS was to change the front-end of an already complete tool, Bico, a prelude generator for the proof assistant Coq. Its original architecture for handling options was the following:

- a Main class file containing methods to parse the options and set their values in a structure (174 lines), and

- an Option enum type containing the options' descriptions and their exact syntax (87 lines).

Option conversion. Since the options were well defined, most of them were easily translated to a CLOPS description.

The first difficulty we came across was with ambiguous arguments. Bico was taking as arguments a directory and an optional list of class file names. Both these arguments had no specific order on the command line and could interleave.

One method of dealing with this would have been to treat both of them as Strings and distinguish between them later in the Java code. This was not an ideal choice because CLOPS has mechanisms for recognizing files, as well as checking their existence and type, and we wanted to use these features.

The solution (Listing 3) we arrived at was to make the directory a mandatory first argument. The CLOPS specification mandated that the directory must exist. The class files were specified as a list of Strings, where the Strings have to be of a specific form (specified by the argumentshape property).

The second difficulty encountered involved the syntax of CLOPS and the authors of the pertaining code had to be consulted. In Bico there are two options that cannot be specified together. In CLOPS it is specified using a validity 
rule, which produces an error if its condition evaluates to true. The solution to this requirement using a validity rule is given in Listing 4. Such rules are expressive but their syntax is obscure for an outside user and the documentation was insufficient at the time. Still, in the Bico case, it is fairly easy to understand: $\{\$($ Map $) \& \& \$($ List $)\}$ checks if the value of the options Map and List are true at the same time; $\$(o)$ expands to the value of $o$ and $\& \&$ is the Java and operator.

Note that the options Map and List default to false, hence they always do have a value.

\section{ARGS::}

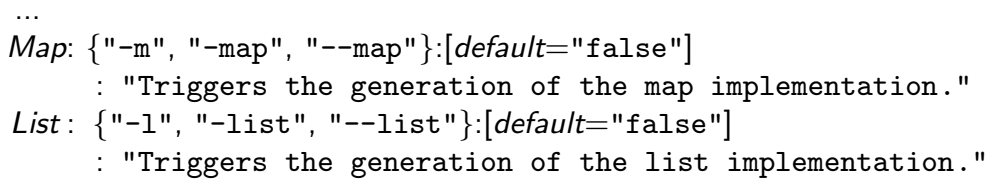

Listing 4. Solution to the second problem

Documentation. The documentation templating was really useful. It facilitates the generation of correct and clear documentation of the options. Some standard templates are provided, but they can be easily modified slightly to fit the requirements of a particular user. For instance, the default templates show for each validity rule two things: the code generated for the test and the explanation. From the end-user point of view, the only item of relevance is the explanation. It was easy to edit the template and remove the code for the test, as the template uses the Velocity language. For many programs it is also necessary to add a copyright statement to all documentation. Again, this can be easily added by some simple template editing.

Statistics. The Main file that uses CLOPS is 134 lines long. The CLOPS description is 47 lines long, for a total of 181 lines of CLOPS-related code to write. Since the original argument-specific code for the program was 261 lines, there was therefore a reduction of 80 lines. In summary the use of CLOPS led to a code reduction of roughly $25 \%$ and adds information that was not originally present, such as proper descriptions of the different arguments.

\subsection{Other CLOPS Usage Examples}

Clarifying arguments handling. The second tool that we used CLOPS on was Mobius' DirectVCGen. It is a generator of verification conditions from annotated Java source files. It uses ESC/Java2 as a front-end to handle the annotations and the Java source. This case is interesting because it has options inherited from ESC/Java2 as well as several options of its own. ESC/Java2 has 
over a hundred options and DirectVCGen actually only uses a few of them, but was not displaying an error message when an unsupported option was provided.

The CLOPS specification has allowed the identification of which arguments were used by DirectVCGen, and what their syntax was. Five arguments were identified, two specific to the DirectVCGen and three inherited from ESC/Java2. Here the aspect of CLOPS that was emphasized was its ability to help provide clear descriptions of the options as well as proper documentation.

The code to handle arguments in the original version was of 118 lines long, and in the new version with CLOPS is 132 lines long (101 lines of Java and 31 lines of CLOPS specification). The main gain here is having a proper description of the options which was not the case in the first version and thus making the program more robust.

Developing with CLOPS. The third tool we have used CLOPS on is LogicSync, a prelude consistency checker for ESC/Java2. The interest in using CLOPS right from the start of development was to be able to properly test the tool even at the early stages and also to be able to easily keep documentation up to date as the argument syntax evolves. Thanks to the experience acquired with the two other examples there were no specific difficulties in writing the CLOPS description. It was completed, together with the integration to the Java program, in less than one hour.

\section{Related Concepts}

A CLOPS-generated parser performs two prominent operations: 1) Processes the given sequence of strings (a command line) and returns a corresponding set of option values. 2) Decides whether the combination of option values is valid or not.

There is a variety of techniques aimed at describing sets of combinations of certain entities. A grammar describes a set of sequences of tokens; such sets are known as languages. A logic formula corresponds to a set of interpretations that satisfy the formula.

In CLOPS we can find reflections of both: the format string defines a regular language of options imposing restrictions on how options are sequenced on the command line; the validity function imposes restrictions on option values.

We should note, however, that grammars are typically used for quite a different purpose than the command line as they typically correspond to complex nested structures that drive compilers, interpreters, etc.

On the other hand, the elements on the command line live quite independently of one another and each corresponds to a wish of the user running the program. Some elements of the command line provide necessary information for the program to carry out its job, such as cp fileSrc fileDest-the copy program hardly can do anything if it does not know which files to copy or where. Some elements of the command line trigger a certain behavior of the program. For instance, the program is will happily run with or without the argument 
-C (column formatting) or the argument -1 (one-per-line formatting). Each of these arguments corresponds to a feature of the program and the command line enables the user to express which features he requires.

In the program $1 \mathbf{s}$, the feature -1 (long listing) is even so popular that users typically alias the invocation $1 \mathrm{~s}-1$ to 11 . This provides us with an alternative view on the program: the original 1 s represents a family of programs and 11 is a member of that family.

This brings us to another relevant domain Software Product Lines (SPL), which studies families of programs [5]. In SPL an individual program is combination of certain features, and a family of programs is a set of feature combinations. The individual features and dependencies between them are captured in feature model 11 .

In SPL terminology, the command line options represent variability of the program in question. In general, variability can be bound at different times, e.g., at compile-time or at run-time [13. In our case, it is always bound at run-time. From the user perspective, however, there is no difference between an alias 11 and a program called 11 (the efficiency issue here is negligible).

Command line options are commonly regarded as something of small importance but we believe that is an underestimation. Deciding which options a particular program supports determines the scope of the variability in the program. The POSIX standard for common command line utilities is an evidence that it is not a trivial task.

In SPL, examining the scope of variability is known as feature oriented domain analysis (FODA) [1]. The variability in FODA is captured in the feature model, which is typically captured as a feature diagram. A feature model corresponds to a CLOPS description as both are variability models.

This discussion brings us back to grammars, in SPL the relation between grammars and feature models is not new [2]. Grammars are particularly useful in approaches where the order of features is important [3].

\section{Related Work}

There is a relatively large number of libraries for processing command line options. We identified three main groups of command line libraries.

The first group consists of libraries that follow the philosophy of the original Unix getopt function (Commons CLI [6] and JSAP [10]). These libraries usually provide only limited capabilities on top of basic getopt. Option values are queried via a Map-like interface and are usually treated as Strings or a particular type explicitly predefined by a getter method name. Libraries from the first group are ignorant of dependencies between options - dependencies must be explicitly handled by the tool developer.

Libraries in the second group recognize options via annotations of variables or methods in the source code (JewelCLI [9] and Args4j [1]). This approach has several advantages to the getopt-like option specification. Types are extracted directly from the source code and therefore the parser automatically checks 
parameter syntax. Unfortunately, libraries in the second group also ignore option interdependencies.

The third group contains libraries that process options externally specified (JCommando [8]). An external specification is compiled into a library, much like in the CLOPS approach.

Table 2 summarizes and compares some of the best known of these libraries with CLOPS in several ways. Libraries are compared based upon their approach, features, and flexibility.

Help extraction is the ability to generate help documentation from the command line specification. Incremental options are options where multiple uses change the state of the option in some way, for example using $-\mathrm{v}$ more than once often increases the level of verbosity. Relations among options are mechanisms to specify dependencies between options (for instance, two options that cannot be used together, an option that can only be used in conjunction with another, etc.).

Some of the libraries allow call-backs during specific stages of a parse (e.g. after an option has been set). Thus, for some of the abilities detailed it is possible for the developer to write extra code to achieve the desired effect. In these cases the libraries do not facilitate the functionality, but do not prevent it either.

Most of the libraries support short and long variants of option aliases. Supporting a list of variants, rather than only a short/long pair, is rather exceptional (JewelCLI). Strict adherence to a finite enumeration of option-alias strings is a limitation in the flexibility of specifying option names. For example, the tail command prints a number of lines from the end of the input, where the exact number is specified as an option of the form -NUMBER. Even if a list of variants is allowed, specifying the aliases for this type of option would be too verbose to be an acceptable approach.

The leading hyphen is used almost exclusively as an option prefix. However, alternative prefixes are often attractive in some situations. For example, the chmod command uses both - and + as prefixes for options and effectively distinguishes when a particular feature is to be enabled or disabled. Another example is the tradition of some Windows command line tools to use slash (/) instead of hyphen (-).

Although it is often useful, the format of a command line is another feature omitted by most libraries. Many tools (including svn, zip, and chown) require a command name early in a command line in order to properly process later options. If there is no support for format within a library, as is nearly uniformly the case, such functionality must be hard-coded.

Dependencies among options are almost always ignored in current command line utilities and, therefore, manual testing the validity of option values is delegated to the user of the library. As mentioned in the Introduction, this leads to overly complicated and potentially buggy code.

As an exception, we have found the JCommando 8] library. JCommando introduces the notion of a command, which is similar to an option except that only one command can be set by a command line. The set method for the 


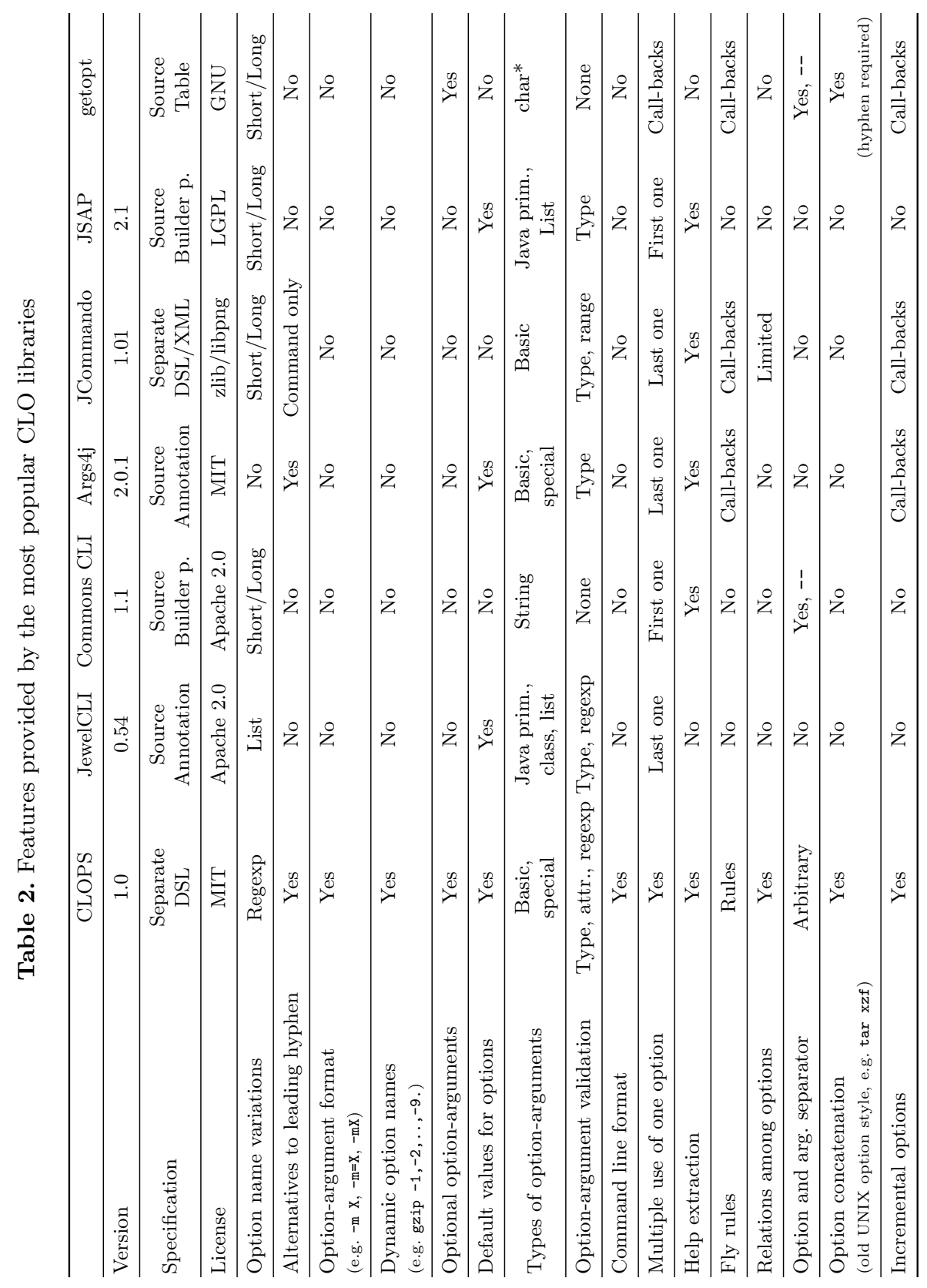


command is called last, after all option set methods. The fallback command ("commandless") is used if no others are set by the user.

A command can specify what options can/should have been set for a valid parse. This is done using a boolean expression over option identifiers. Each identifier will evaluate to true or false at runtime according to whether that option was set or not. The boolean expression for the provided command is evaluated when that command is set, and if it evaluates to false an error is produced. This is a much simpler form of validity check than we allow. In particular, there is no way to relate options to each other outside the context of a command, nor is there a way to use option values in the test.

Surprisingly, many libraries do not provide a mechanism to enable a developer to specify, for example, filenames that start with a hyphen as any string starting with a hyphen is assumed to be an option. UNIX getopt solves this problem using the special string --, which is modeled in a CLOPS specification by the simple aforementioned format expression (see Section 5.1).

Feature modeling 11] has a goal similar to that of our DSL. A feature model explicitly captures the variability and commonality of a program [5]. In fact, one can imagine a program as a family of programs whose members correspond to the possible configurations of the feature model, as expressed via command line options. Whereas feature models target various types of variabilities at design- and compile-time, command line options represent variability resolved at the runtime.

\section{Conclusions and Future Work}

Many command line tools solve the command line parsing problem using custom code, sometimes relying on a little help from specialized libraries. "A DSL is viewed as the final and most mature phase of the evolution of object-oriented application frameworks," according to Deursen et al 14. CLOPS aims to succeed and supersede existing libraries in this regard. Combining an analysis of the set of existing solutions with the identification and refinement of the domain-specific concepts of command line options, a minimized conceptual framework is defined. Consequently, the syntax of the DSL is concise and self-documenting, reflecting exactly this conceptual design, which lends itself to a gradual learning curve. Additionally, while the DSL is simple enough to formalize and is easy to use, it is also powerful enough to cover all command line conventions of which we are aware.

The implementation is done classically, by writing a compiler that generates Java source code. From our experience of reimplementing the interface of standard UNIX tools and implementing the interface of some other tools, CLOPS increases a programmer's productivity, though the quantification of such is the subject of further work.

Some aspects are painfully missing from the current implementation. One compelling aspect of many DSLs is that consistency checks are accomplished at a higher level of abstraction. One such consistency check for CLOPS is the ability to statically analyze a CLOPS description to guarantee that the generated command line parser compiles and does not fail in certain ways. For example, the 
CLOPS system might perform type-checking instead of deferring such to the Java compiler. Creators of other DSLs found that early type-checking is sometimes more precise typechecking [14]. Another potential static check is ensuring regular expressions used to match options that can legally appear in the same parsing state are disjoint. This would ensure that the Matched set contains at most one element. (Of course, the parser would still fail at runtime when Matched is empty.) Finally, we require, but do not check, that the expressions used in rules do not have side-effects. Existing research on containing side-effects in languages like Java will prove useful [7,4].

Other parts are missing, but are less troublesome. A hassle that accompanies code generation is a more complicated build process. This annoyance is sometimes avoided by generating the parser at runtime. Of course, such an architecture change may hurt performance, but, for many command line parsing tools, we expect performance to be less important than ease of use.

In various flavors of UNIX there are many implementations of common command line POSIX utilities such as chmod and 1s. After describing a single command line interface for a given tool across a suite of implementations one might generate command line strings automatically for system-level testing. Such work has been done in a limited form already here at UCD for the OpenCVS project.

Finally, some of this work is applicable in the context of tools with graphical, rather than textual, interfaces. GUI programs often store their settings in a preferences store that is read upon start-up, and many such programs provide a 'wizard' (e.g., a preferences pane) to populate the settings file. While it requires is no stretch of the imagination to envisage the CLOPS framework generating the wizards and the parser for such GUI programs, important details need to be clarified before this step is made.

\section{Acknowledgments}

This work is funded, in part, by Science Foundation Ireland under grant number 03/CE2/I303-1 to "Lero: The Irish Software Engineering Research Centre", and by the Information Society Technologies programme of the European Commission, Future and Emerging Technologies under the IST-2005-015905 MOBIUS project, and by an EMBARK Scholarship from the Irish Research Council in Science, Engineering and Technology. Viliam Holub is supported by the IRCSET Embark Postdoctoral Fellowship Scheme. The article contains only the authors' views and the Community is not liable for any use that may be made of the information therein.

\section{References}

1. Args4j, http://args4j.dev.java.net/

2. Batory, D.: Feature models, grammars, and propositional formulas. In: Obbink, H., Pohl, K. (eds.) SPLC 2005. LNCS, vol. 3714, pp. 7-20. Springer, Heidelberg (2005) 
3. Batory, D., O'Malley, S.: The design and implementation of hierarchical software systems with reusable components. ACM Transactions on Software Engineering and Methodology (1992)

4. Clarke, D.G., Potter, J.M., Noble, J.: Ownership types for flexible alias protection. SIGPLAN Not. 33(10), 48-64 (1998)

5. Clements, P., Northrop, L.: Software Product Lines: Practices and Patterns. Addison-Wesley Publishing Company, Reading (2002)

6. The Apache Commons CLI library, http://commons.apache.org/cli/

7. Darvas, A., Muller, P.: Reasoning about method calls in JML specifications. In: Formal Techniques for Java-like Programs (2005)

8. JCommando: Java command-line parser, http://jcommando.sourceforge.net/

9. JewelCLI, http://jewelcli.sourceforge.net/

10. JSAP: The Java Simple Argument Parser, http://www .martiansoftware.com/jsap/

11. Kang, K.C., Cohen, S.G., Hess, J.A., Novak, W.E., Spencer Peterson, A.: Featureoriented domain analysis (FODA), feasibility study. Technical Report CMU/SEI90-TR-021, SEI, Carnegie Mellon University (November 1990)

12. Leavens, G.T., Baker, A.L., Ruby, C.: JML: A Notation for Detailed Design. In: Behavioral Specifications of Business and Systems, pp. 175-188. Kluwer Academic Publishing, Dordrecht (1999)

13. Svahnberg, M., Van Gurp, J., Bosch, J.: A taxonomy of variability realization techniques. Software-Practice and Experience 35(8), 705-754 (2005)

14. van Deursen, A., Klint, P., Visser, J.: Domain-specific languages: an annotated bibliography. SIGPLAN Not. 35(6), 26-36 (2000)

15. The Apache Velocity Project, http://velocity.apache.org/ 